Physical Activity and Nutrition I 2021;25(2):008-014

Received: 2021/04/12, Revised: 2021/06/07, Accepted: 2021/06/24, Published: 2021/06/30 @2021 Kevin Boldt et al.; Licence Physical Activity and Nutrition. This is an open access article distributed under the terms of the creative commons attribution license (https:/l creativecommons.org/licenses/by-nc/2.0/), which permits unrestricted use, distribution, and reproduction in any medium, provided the orginal work is properly cited.

${ }^{*}$ Corresponding author : Kevin Boldt, Ph.D.

Human Performance Laboratory, Faculty of Kinesiology, University of Calgary, 2500 University Drive, Calgary, T2N $1 \mathrm{~N} 4$, Canada

Tel: +1-226-377-6696

E-mail: krboldt@ucalgary.ca

@2021 The Korean Society for Exercise Nutrition

\section{Consumption of a high-fat-high- sucrose diet partly diminishes mechanical and structural adaptations of cardiac muscle following resistance training}

\author{
Kevin Boldt ${ }^{1^{*}}$ / Stela Mattiello ${ }^{2}$ / Venus Joumaa ${ }^{1}$ / \\ Jeannine Turnbull ${ }^{3}$ / Paul W.M. Fedak ${ }^{3}$ / Walter Herzog ${ }^{1,4}$ \\ 1. Human Performance Laboratory, University of Calgary, Calgary, Canada \\ 2. Department of Physical Therapy, Center of Biological and Health Sciences, Federal University of São \\ Carlos, São Carlos, Brazil \\ 3. Health Centre, Federal University of Santa Catarina, Florianopolis, Brazil \\ 4. Department of Cardiac Sciences, University of Calgary, Calgary, Canada
}

\section{INTRODUCTION}

The purpose of this study was to investigate thigh-fat high-sucrose (HFHS) diet on previously reported adaptations of cardiac morphological and contractile properties to resistance training.

[Methods] Twelve-week-old rats participated in 12-weeks of resistance exercise training and consumed an HFHS diet. Echocardiography and skinned cardiac muscle fiber bundle testing were performed to determine the structural and mechanical adaptations.

[Results] Compared to chow-fed sedentary animals, both HFHS- and chow-fed resistance-trained animals had thicker left ventricular walls. Isolated trabecular fiber bundles from chow-fed resistance-trained animals had greater force output, shortening velocities, and calcium sensitivities than those of chow-fed sedentary controls. However, trabeculae from the HFHS resistance-trained animals had greater force output but no change in unloaded shortening velocity or calcium sensitivity than those of the chow-fed sedentary group animals.

[Conclusion] Resistance exercise training led to positive structural and mechanical adaptations of the heart, which were partly offset by the HFHS diet.

[Key words] obesity, high-fat high-sucrose diet, cardiac adaptations, resistance exercise, skinned fibers, echocardiography

[Abbreviations]

HFHS: High-fat-high-sucrose

CVD: Cardiovascular disease

BMl: Body mass index

EDV: End diastolic volume

ESV: End systolic volume

EF: Ejection fraction

LV: Left ventricle

LVID: Left ventricle internal diameter

LVAW: Left ventricle anterior wall

LVPW: Left ventricle posterior wall

$\mathrm{pCa}_{50}$ : Calcium sensitivity yielding half of maximal force

$\mathrm{nH}$ : Crossbridge cooperativity
There are significant links between cardiovascular disease (CVD) and obesity ${ }^{1}$. In humans, each increase of $5 \mathrm{~kg} / \mathrm{m}^{2}$ in body mass index (BMI) results in a 30\% increase in CVD risk ${ }^{2}$. Similarly, individuals with a BMI $\geq 35 \mathrm{~kg} / \mathrm{m}^{2}$ have a 2-3-fold greater risk of death from CVD than individuals with a normal BMI $\left(18.5-24.9 \mathrm{~kg} / \mathrm{m}^{2}\right)^{3}$. The deleterious effect of obesity on the heart is further exacerbated by common comorbidities associated with obesity, which have also been independently linked to cardiovascular disease ${ }^{1}$.

Recently, Boldt et al. ${ }^{4}$ exposed post-weaning (3-week-old) rats to a high-fat high-sucrose (HFHS) diet for 14 weeks as they matured through juvenile development. Isolated muscle tissue from the hearts of animals exposed to an obesogenic diet had lower unloaded shortening velocities and calcium sensitivities than those in chow-fed control animals. In a follow-up study, in which 12-week-old rats were exposed to the same HFHS diet for 24 weeks, HFHS-fed rats had significantly lower shortening velocity and tended to have lower calcium sensitivity than those of chow-fed control animals ${ }^{5}$. Similar findings have also been reported for lower shortening velocities ${ }^{6-8}$ and depressed calcium handling ${ }^{7,8}$ following HFHS diet-induced obesity in mice and rats.

Exercise has been shown to have differential effects on cardiac contractile properties depending on the exercise modality. Aerobic exercise has been shown to be associated with greater shortening velocity and calcium sensitivity in isolated cardiac muscle than those of sedentary controls $^{9-13}$. In response to resistance exercise, isolated cardiac muscle preparations have been associated with greater active force production, shortening velocity, and calcium sensitivity than those in sedentary controls $^{5,14}$. Structurally, aerobic exercise has been shown to result in greater ventricular volume and proportionally thicker walls (eccentric hypertrophy), while resistance-trained hearts have been characterized by thicker walls but similar volumes (concentric hypertrophy) compared to hearts from sedentary individuals ${ }^{15-18}$. 
When combined, HFHS diets have been shown to inhibit the positive adaptations of aerobic exercise. For example, when 12-week-old rats were exposed to 24 weeks of an HFHS diet and participated in aerobic exercise from weeks 13 to 24 , the unloaded shortening velocity and calcium sensitivity were not different from those of chow-fed sedentary animals ${ }^{13}$. Thus, consumption of an HFHS diet appears to inhibit the positive adaptations that result from aerobic exercise. Although resistance exercise training has been shown to improve the mechanical function of the heart, it is not known how cardiac mechanical properties respond to resistance training and simultaneous exposure to an HFHS diet. The purpose of this study was to investigate the effects of an HFHS diet on the adaptations of cardiac morphological and contractile properties typically observed with resistance training. The cardiac morphological and mechanical properties of HFHS-fed and resistance-trained animals were compared to previously reported data ${ }^{13}$ from chow-fed resistance-trained and sedentary animals.

\section{METHODS}

\section{Animals and diets}

Twelve-week-old male Sprague-Dawley rats were fed a HFHS diet and participated in a 12-week resistance training program (HFHS+Res, $n=7$ ). The HFHS diet (Dyets\#102412) comprised of $20 \%$ protein, $20 \%$ fat, and $50 \%$ carbohydrates ( $>97 \%$ of carbohydrates from sucrose), and 10\% fiber and moisture, and has previously been shown to result in obesity ${ }^{19,20}$. Data from the HFHS+Res animals were compared to data from a previous training study in which twelve-weekold male Sprague-Dawley rats were fed a chow diet and were either sedentary (Chow + Sed, $n=5$ ) or participated in 12 weeks of resistance exercise (Chow+Res, $n=6)^{13}$. The chow diet consisted of $25 \%$ protein, $5 \%$ fat, and $48 \%$ carbohydrates ( $3 \%$ carbohydrates from sucrose), and $22 \%$ fiber and moisture (Dyets\#5001).

Animals were housed individually at $21^{\circ} \mathrm{C}$ in a 12-h:12-h light-dark cycle and had access to food and water ad libitum. All study protocols were approved by the University of Calgary Animal Care Committee and conformed to the Guide for the Care and Use of Laboratory Animals.

\section{Resistance training}

Resistance training consisted of a progressive weighted ladder climbing exercise $3 \mathrm{~d}$ per week ${ }^{21,22-24}$. Briefly, rats climbed a $1-\mathrm{m}$ ladder inclined at $80^{\circ}$ to the horizontal with a weight secured to the base of their tails. The initial load for each animal was $50 \%$ of their body mass. After each successful ladder climb, rats were given a 2-min rest period, and the load was increased by $30 \mathrm{~g}$. The rats continued to climb with progressively heavier loads until they were unable to complete the ladder climb, and the load for the last successful ladder climb was designated as the maximum load. Each subsequent training session consisted of climbing with $50 \%, 75 \%, 90 \%$, and $100 \%$ of the previous maximum training load. During each subsequent climb after 100\%, an additional $30 \mathrm{~g}$ was added until the rat could not overcome the load, and the new maximum training load was reached. The weekly maximum load was defined as the greatest load carried each week during training.

\section{Body mass and body composition}

At the end of the 12-week intervention period, rats were anesthetized using isoflurane, and their body mass was measured using a small animal weight scale. Body fat percentage was then measured using dual energy X-ray absorptiometry with the animals in the supine position using software for small animals (GE Medical Systems Lunar, Madison, USA).

\section{Cardiac structural and functional adaptation}

Echocardiographic evaluation was performed using an Esaote MyLab30 Gold Cardiovascular Ultrasound system (Canadian Veterinary Imaging, Georgetown Ontario). Rats were anesthetized with isoflurane and placed in the dorsal decubitus position, and the ventral thoracic area was shaved. Two-dimensional orthogonal long axis four- and two-chamber images were obtained ${ }^{25}$. End diastolic volume (EDV), end systolic volume (ESV), and ejection fraction (EF) were calculated from three consecutive cardiac cycles by tracing the endocardial border in end-diastole and end-systole using the Simpson biplane method ${ }^{25}$. Left ventricular (LV) internal diameter (LVID) and thickness of the anterior and posterior LV walls were determined using M-mode imaging during systole (LVIDs, LVAWs, LVPWs) and diastole (LVIDd, LVAWd, LVPWd).

\section{Mechanical testing}

Mechanical testing was conducted on skinned cardiac trabeculae muscle, as described in detail previously ${ }^{5}$. Briefly, skinned fiber bundles (approximately 100-300 $\mu \mathrm{m}$ in width and 1000-2000 $\mu \mathrm{m}$ in length) were suspended between a length controller (Aurora Scientific Inc., Model 308, Ontario, Canada) and force transducer (Aurora Scientific Inc., Model 400A, Ontario, Canada), thereby allowing for simultaneous control of myocyte length and measurement of force. All experiments were performed at approximately $15^{\circ} \mathrm{C}$ at an average resting sarcomere length of $2.2 \mu \mathrm{m}$. Following a 3-min rest period to allow for any stress relaxation, passive (resting) force was measured, and the length and width of the specimens were measured to calculate the cross-sectional area of the sample. Two skinned samples were tested from each heart, and the data were pooled for statistical analysis.

Fiber bundles were maximally activated, and maximal active stress was calculated as the difference between the total force and resting passive force preceding the contraction, normalized to the cross-sectional area.

The maximal unloaded shortening velocity was determined using the slack test, initially described by Edman ${ }^{26}$. The fiber bundles were maximally activated and rapidly shortened (in $2 \mathrm{~ms}$ ) by $10 \%(\Delta \mathrm{L})$ of the length of the sample, and the time from the onset of the quick shortening step until the onset of force redevelopment $(\Delta t)$ was measured. 
This procedure was repeated for $\Delta \mathrm{L}$ values of $11 \%, 12 \%$, and $13 \%$ of the sample length. The slope of the linear approximation between $\Delta \mathrm{L}$ and $\Delta \mathrm{t}$ was used to determine the unloaded (maximal) shortening velocity.

The sensitivity of the muscle to calcium was determined by establishing the force-pCa curve from $\mathrm{pCa} 7.0,6.8,6.6$, $6.4,6.2,6.0,5.8,5.4$, and 4.2 , by approximating the data using a least-squares regression for the Hill equation in SigmaPlot 13. Calcium sensitivity was obtained and quantitatively compared between samples by determining the $\mathrm{pCa}$ that yielded half of the maximal tension $\left(\mathrm{pCa}_{50}\right)$ and crossbridge cooperativity $(\mathrm{nH})^{27}$.

\section{Analysis}

Kruskal-Wallis tests were used to determine statistical significance with pairwise comparisons to determine the differences between groups when specified. Statistical significance was accepted at $\mathrm{p}<0.05$.

\section{RESULTS}

\section{Resistance exercise training}

On average, the weekly maximum loads carried by the resistance-trained animals increased from baseline by 2.63and 2.32-fold for the Chow+Res and HFHS+Res animals, respectively. There were no differences in the magnitude of adaptation between the two groups $(p=0.475)$. At the end of the 12 weeks, there were no differences in body mass $(p=0.366)$ or body fat percentage $(p=0.062)$ between groups (Table 1).

\section{Cardiac structural and functional adaptation}

The structural data obtained via echocardiography are shown in Figure 1. There were no differences between groups for heart volume (EDV [ $\mathrm{p}=0.797]$, $\mathrm{ESV}[\mathrm{p}=0.847$ ], LVIDd [ $p=0.179]$, LVIDs $[p=0.248])$ or EF $(p=0.104)$. Hearts from sedentary animals (Chow + Sed) had significantly thinner anterior LV walls (LVAWd and LVAWs) than both the chow-fed (Chow+Res, $\mathrm{p}<0.036)$ and HFHS-fed $($ HFHS + Res, $\mathrm{p}<0.050)$ resistance-trained animals. Similarly, posterior LV walls (LVPWd) were significantly thinner in the Chow+Sed group than in the Chow+Res group $(p=0.010)$, but were not different from the HFHS+Res animals $(p=0.160)$. There were no significant differences in LVPWs between groups $(\mathrm{p}=0.127)$. There were no significant differences in structure between the Chow + Res and
HFHS+Res animals.

\section{Mechanical testing}

Active stress was greater in samples from animals in the Chow + Res $(p=0.022)$ and HFHS+Res $(p=0.030)$ groups than in samples from the control sedentary animals (Figure 2A). The Chow+Res and HFHS+Res groups were not significantly different $(\mathrm{p}=0.832)$.

The maximum shortening velocity, determined using the slack test, was significantly higher in the Chow+Res animals than in both the sedentary $(\mathrm{p}=0.002)$ and HFHS+Res $(\mathrm{p}=0.001)$ animals, but did not differ between the Chow + Sed and HFHS+Res animals $(\mathrm{p}=0.910)$ (Figure 2B).

Calcium sensitivity ( $\mathrm{pCa}_{50}$ values) was significantly greater in the Chow+Res group than in the Chow + Sed $(\mathrm{p}=0.018)$ and HFHS + Res $(\mathrm{p}=0.001)$ group animals (Figure $2 \mathrm{C}$ ), but was not different between the Chow+Sed and HFHS + Res groups $(p=0.389)$. There were no significant differences in $\mathrm{nH}$ between groups $(\mathrm{p}=0.550)$ (Figure 2D).

\section{DISCUSSION}

The purpose of this study was to investigate the effects of an HFHS diet on the adaptations of cardiac morphological and contractile properties to resistance training. The main findings were that both HFHS and chow-fed resistance-trained animals had thicker ventricular walls than those of sedentary controls (concentric hypertrophy), but they did not differ from each other. Furthermore, while the chow-fed resistance-trained animals had greater force production, shortening velocity, and calcium sensitivity than those of sedentary animals, the HFHS resistance-trained animals had greater force production but similar maximal unloaded shortening velocity and calcium sensitivity compared to those of sedentary animals.

\section{Resistance exercise training}

The ladder-climbing protocol used in this study has been shown to increase strength ${ }^{21}$. Climbing performance increased for both resistance-trained groups by about 2.5 -fold, suggesting that the training stimulus was effective in improving muscular strength and was not affected by the diet.

\section{Cardiac structural adaptations}

The expected structural adaptations to resistance exercises are controversial. Adaptations to resistance training

Table 1. Descriptive end-point data. Data displayed are medians (IQR). * indicates a significant change from baseline $(p<0.05)$

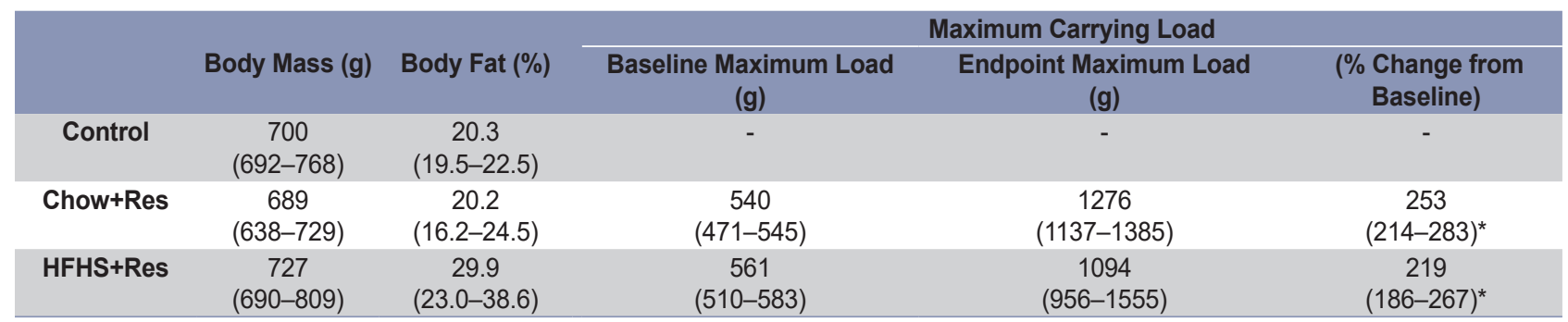



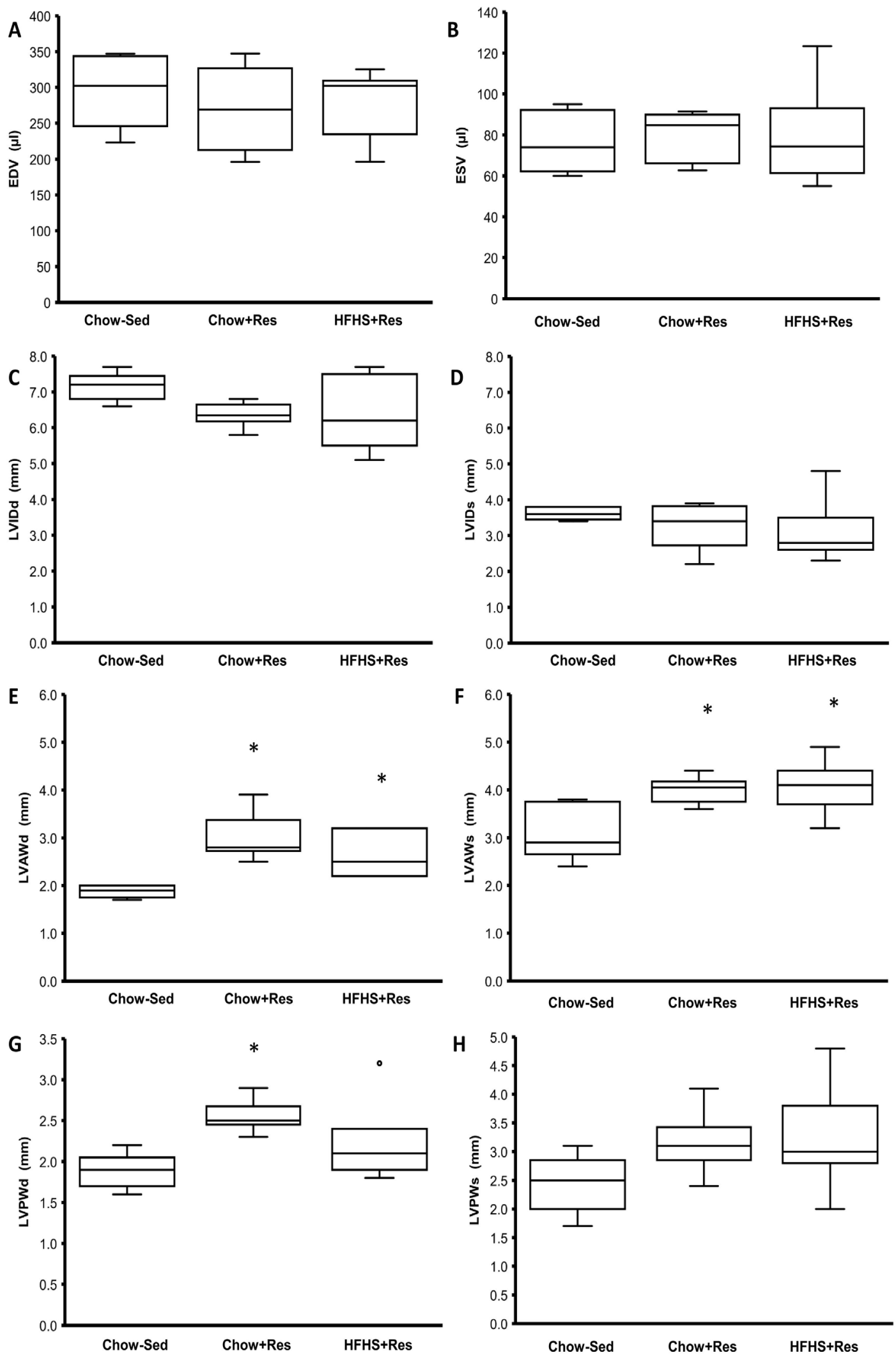

Figure 1. Cardiac structural data from echocardiogram. * indicates a significant difference from the Chow+Sed group animals. 

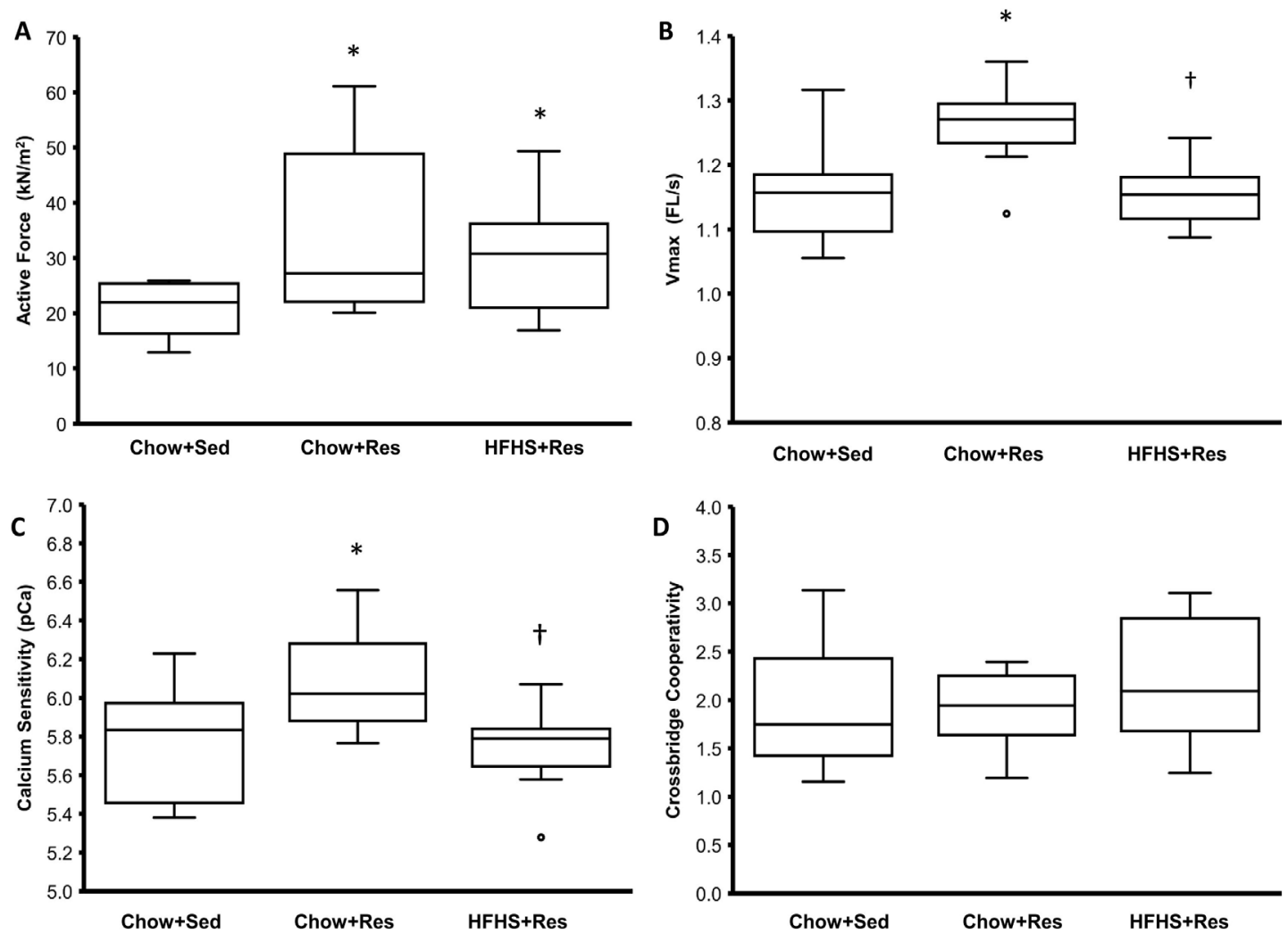

Figure 2. Mechanical data. A, active stress; B, unloaded shortening velocity; C, calcium sensitivity ( $p$ Ca50); and D, cross bridge cooperativity ( $\mathrm{nH}$ )) from skinned trabeculae preparations. * indicates a significant difference from the Chow+Sed group animals. $=, \dagger$ indicates a significant difference from the Chow+Res group animals $(p<0.05)$

have generally been characterized by concentric hypertrophy (thickening of muscular walls with no change in stroke volume $)^{15,17}$. However, these findings have not been unanimously supported ${ }^{18,28}$. Our previous findings from resistance-trained animals support concentric hypertrophy of the heart. We observed concentric hypertrophy in the HFHS+Res group rats in this study, and the magnitude of adaptation was similar for the Chow+Res and HFHS+Res group animals.

Consumption of HFHS diets has also been shown to result in thickening of the LV walls ${ }^{29}$. Leopoldo et al. ${ }^{30}$ exposed Wistar rats to 15 weeks of either a chow or high-fat diet. They observed no differences in end diastolic volume, but animals in the high-fat diet group had thicker ventricular walls and greater LV mass. Since consumption of a high-fat diet and resistance training independently leads to thickening of cardiac muscular walls, it is difficult to interpret our findings. More research should be conducted to determine the differences between pathological hypertrophy resulting from obesity and consumption of an HFHS diet, and adaptive hypertrophy resulting from exercise. However, with consumption of a high-fat diet, thickening of the muscular walls is also associated with decrements in contractile function $^{29,30}$. In the present study, we observed improvements in contractile function with resistance training, but these improvements were partly diminished in the animals exposed to the HFHS diet.

\section{Cardiac mechanical adaptations}

Aerobic exercise has been shown to result in increased shortening velocity and calcium sensitivity, but unaltered maximum force production in cardiac tissue ${ }^{9,11,12}$. Conversely, consumption of an HFHS diet results in decreased shortening velocity and calcium sensitivity ${ }^{4,13}$. Muscle isolated from the hearts of rats exposed to 24 weeks of an HFHS diet had lower shortening velocity and calcium sensitivity than chow-fed controls ${ }^{13}$. However, when the HFHS diet was combined with aerobic exercise, the shortening velocity and calcium sensitivity were comparable to those observed in sedentary chow-fed controls. The effects of exercise and an HFHS diet on cardiac contractile properties seem to neutralize each other.

Following resistance training, cardiac muscle has been shown to have increased force production, shortening velocity, and calcium sensitivity ${ }^{13,14}$. In the present study, resistance training combined with an HFHS diet resulted in unloaded shortening velocities and calcium sensitivities that were similar to those obtained from chow-fed sedentary animals. Similar to previous findings in rats exposed to aerobic exercise, the HFHS diet combined with resistance training offset the positive adaptations typically associated with exercise. In contrast to unloaded shortening velocity and calcium sensitivity, active force production was not affected by the HFHS diet ${ }^{4,5,29}$, and ladder climbing ability improved with resistance exercise training. 
Regardless of diet, participation in resistance training leads to positive adaptations of cardiac tissue. However, consumption of an HFHS diet reduces the positive adaptions typically observed in the heart muscle with resistance training.

\section{REFERENCES}

1. Kenchaiah S, Evans JC, Levy D, Wilson PW, Benjamin EJ, Larson MG, Kannel WB, Vasan RS. Obesity and the risk of heart failure. N Engl J Med. 2002;347:305-13.

2. Selmer R, Tverdal A. Body mass index and cardiovascular mortality at different levels of blood pressure: a prospective study of Norwegian men and women. J Epidemiol Community Health. 1995;49:265-70.

3. Calle EE, Thun MJ, Petrelli JM, Rodriguez C, Heath Jr CW. Bodymass index and mortality in a prospective cohort of US adults. $N$ Engl J Med. 1999;341:1097-105.

4. Boldt K, MacDonald G, Joumaa V, Herzog W. Mechanical adaptations of skinned cardiac muscle in response to dietary-induced obesity during adolescence in rats. Appl Physiol Nutr Metab. 2020;45:893-901.

5. Boldt K, Rios JL, Joumaa V, Herzog W. Mechanical function of cardiac fibre bundles is partly protected by exercise in response to diet-induced obesity in rats. Appl Physiol Nutr Metab. 2021;46-54.

6. Li S-Y, Yang X, Ceylan-Isik AF, Du M, Sreejayan N, Ren J. Cardiac contractile dysfunction in Lep/Lep obesity is accompanied by NADPH oxidase activation, oxidative modification of sarco (endo) plasmic reticulum Ca 2+-ATPase and myosin heavy chain isozyme switch. Diabetologia. 2006;49:1434-46.

7. Dong F, Zhang X, Yang X, Esberg LB, Yang H, Zhang Z, Culver $B$, Ren J. Impaired cardiac contractile function in ventricular myocytes from leptin-deficient ob/ob obese mice. J Endocrinol. 2006;188:25-36.

8. Relling DP, Esberg LB, Fang CX, Johnson WT, Murphy EJ, Carlson EC, Saari JT, Ren J. High-fat diet-induced juvenile obesity leads to cardiomyocyte dysfunction and upregulation of Foxo3a transcription factor independent of lipotoxicity and apoptosis. $J$ Hypertens. 2006;24:549-61.

9. Diffee GM, Chung E. Altered single cell force-velocity and power properties in exercise-trained rat myocardium. J Appl Physiol. 2003;94:1941-8.

10. Chung E, Diffee GM. Moderate intensity, but not high intensity, treadmill exercise training alters power output properties in myocardium from aged rats. J Gerontol A Biol Sci Med Sci. 2012; 67:1178-87

11. Diffee GM, Seversen EA, Titus MM. Exercise training increases the $\mathrm{Ca} 2+$ sensitivity of tension in rat cardiac myocytes. J Appl Physiol. 2001;91:309-15.

12. Diffee GM, Nagle DF. Exercise training alters length dependence of contractile properties in rat myocardium. J Appl Physiol. 2003;94:1137-44.

13. Boldt K, Joumaa V, Turnbull J, Fedak PWM, Herzog W. Mechanical and structural remodeling of cardiac muscle following aerobic and resistance exercise training in rats. Med Sci Sports Exerc. 2021;Published ahead of print.

14. Pinter R de CCE, Padilha AS, De Oliveira EM, Vassallo DV, de
Fúcio Lizardo JH. Cardiovascular adaptive responses in rats submitted to moderate resistance training. Eur J Appl Physiol. 2008;103:605-13.

15. Morganroth J, Maron BJ, Henry WL, Epstein SE. Comparative left ventricular dimensions in trained athletes. Ann Intern Med. 1975;82:521-4.

16. Spence AL, Naylor LH, Carter HH, Buck CL, Dembo L, Murray $\mathrm{CP}$, Watson $\mathrm{P}$, Oxborough $\mathrm{D}$, George KP, Green DJ. A prospective randomised longitudinal MRI study of left ventricular adaptation to endurance and resistance exercise training in humans. J Phys. 2011;589:5443-52.

17. Baggish AL, Wang F, Weiner RB, Elinoff JM, Tournoux F, Boland A, Picard MH, Hutter AM, Wood MJ. Training-specific changes in cardiac structure and function: a prospective and longitudinal assessment of competitive athletes. J Appl Physiol. 2008;104:1121-8.

18. Utomi V, Oxborough D, Whyte GP, Somauroo J, Sharma S, Shave R, Atkinson G, George K. Systematic review and meta-analysis of training mode, imaging modality and body size influences on the morphology and function of the male athlete's heart. Heart. 2013;99:1727-33.

19. Rios JL, Bomhof MR, Reimer RA, Hart DA, Collins KH, Herzog $W$. Protective effect of prebiotic and exercise intervention on knee health in a rat model of diet-induced obesity. Sci Rep. 2019;9:3893.

20. Collins KH, Hart DA, Reimer RA, Seerattan RA, Waters-Banker C, Sibole SC, Herzog W. High-fat high-sucrose diet leads to dynamic structural and inflammatory alterations in the rat vastus lateralis muscle. J Orthop Res. 2016;34:2069-78.

21. Hornberger Jr TA, Farrar RP. Physiological hypertrophy of the FHL muscle following 8 weeks of progressive resistance exercise in the rat. Can J Appl Phys. 2004;29:16-31.

22. Kim K, Ahn N, Jung S, Park S. Effects of intermittent ladder-climbing exercise training on mitochondrial biogenesis and endoplasmic reticulum stress of the cardiac muscle in obese middle-aged rats. Korean J Physiol Pharmacol. 2017;21:633.

23. Souza MVC, Leite RD, de Souza Lino AD, de Cássia Marqueti R, Bernardes CF, de Araújo HSS, Bouskela E, Shiguemoto GE, Andrade Perez SE, Kraemer-Aguiar LG. Resistance training improves body composition and increases matrix metalloproteinase 2 activity in biceps and gastrocnemius muscles of diet-induced obese rats. Clinics. 2014;69:265-70.

24. Neto WK, Silva WDA, Ciena AP, Anaruma CA, Gama EF. Vertical climbing for rodent resistance training: a discussion about training parameters. Int J Sports Sci. 2016;6:36-49.

25. Schiller NB, Shah PM, Crawford M, DeMaria A, Devereux R, Feigenbaum H, Gutgesell H, Reichek N, Sahm D, Schnittger I, Silverman NH, Tajik AJ. Recommendations for quantitation of the left ventricle by two-dimensional echocardiography. J Am Soc Echocardiogr. 1989;2:358-67.

26. Edman KA. The velocity of unloaded shortening and its relation to sarcomere length and isometric force in vertebrate muscle fibres. J Phys. 1979;291:143-59.

27. Danieli-Betto D, Betto R, Midrio M. Calcium sensitivity and myofibrillar protein isoforms of rat skinned skeletal muscle fibres. Pflügers Arch. 1990;417:303-8.

28. Haykowsky MJ, Syrotuik DG, Taylor DA, Bell GJ. The effect of high-intensity rowing and combined strength and endurance training on left ventricular systolic function and morphology. Int $J$ 
Sports Med. 2007;28:488-94.

29. Abel ED, Litwin SE, Sweeney G. Cardiac remodeling in obesity. Physiol Rev. 2008;88:389-419.

30. Leopoldo AS, Sugizaki MM, Lima-Leopoldo AP, do Nascimento AF, Luvizotto R de AM, de Campos DHS, Okoshi K, Pai-Silva MD, Padovani CR, Cicogna AC. Cardiac remodeling in a rat model of diet-induced obesity. Can J Cardiol. 2010;26:423-9. 